\title{
Uso de macroinvertebrados acuáticos como sistema de evaluación de las lagunas de estabilización El Salguero (Colombia)
}

\author{
Julio C. Nuñez y Pedro J. Fragoso-Castilla* \\ Universidad Popular del Cesar, Grupo de Investigación Parasitología Agroecología Milenio, Valledupar, Cesar - \\ Colombia (correo-e: pedrofragozo@unicesar.edu.co; julio.nunez2901@hotmail.com).
}

* Autor a quien debe ser dirigida la correspondencia.

Recibido Ene. 7, 2020; Aceptado Mar. 10, 2020; Versión final Abr. 2, 2020, Publicado Jun. 2020

\begin{abstract}
Resumen
El objetivo de este trabajo fue evaluar la eficiencia del sistema de lagunas de estabilización El Salguero mediante el uso de macroinvertebrados acuáticos. Se aplicó del índice BMWP/Col (adaptado para Colombia) y los índices ecológicos de diversidad alfa y beta. Se colectaron macroinvertebrados acuáticos en las lagunas anaeróbicas, facultativas y de maduración. Los 2619 individuos colectados estan distribuidos en tres phylum, tres Clases, cinco Órdenes y 14 Familias. Los resultados obtenidos demuestran la dominancia del orden Díptera. El índice BMWP/Col indica que existen diferencias entre la calidad de agua en las diferentes lagunas de estabilización. Los índices ecológicos alfa y beta mostraron valores de diversidad entre medio y bajo y asociaciones entre las diferentes familias de taxones. En conclusión, la mayor diversidad de los macroinvertebrados acuáticos se obtuvo en las lagunas de maduración siendo el orden Diptera los que tienen mayor presencia de individuos en cada fase de tratamiento.
\end{abstract}

Palabras clave: lagunas de estabilización; contaminación; macroinvertebrados acuáticos; calidad del agua; $\mathrm{BMWP} / \mathrm{Col}$

\section{Use of aquatic macroinvertebrates as an evaluation system for stabilizing El Salguero lagoon (Colombia)}

\begin{abstract}
The objective of this study was to evaluate the efficiency of the El Salguero stabilization lagoon system by examining aquatic macroinvertebrates. The BMWP/Col index adapted for Colombia was applied as well as the ecological indexes of alpha and beta diversity. Aquatic macroinvertebrates were collected from anaerobic, facultative, and maturation lagoons. The 2619 individuals collected were distributed in three phyla, three classes, five orders, and 14 families. The order Diptera was the most dominant. The BMWP/Col index showed differences in water quality between the different stabilization ponds examined. The ecological indexes alpha and beta showed diversity values ranging from medium to low and showed the associations and distributions of taxa families. In conclusion, the majority of the aquatic macroinvertebrate diversity was obtained from maturation lagoons and the order Diptera was the most prevalent in number of individuals at each treatment phase.
\end{abstract}

Keywords: stabilization ponds; pollution; aquatic macroinvertebrates; water quality; BMWP/Col 


\section{INTRODUCCION}

Las lagunas de estabilización son sistemas de tratamiento pasivos muy utilizados para remediar efluentes líquidos, domésticos, agrícolas e industriales Jimoh et al., (2019), utilizan la capacidad mutualista de los microorganismos para producir efluentes altamente purificado y reutilizables, similares a las obtenidas por sistemas convencionales Mambo et al., (2014) a un costo muy inferior Spellman, (2009). Las lagunas de estabilización son usualmente el más apropiado método de tratamiento de aguas residuales de origen doméstico y municipal en países en vías de desarrollo; son opciones de bajo costo, que requieren poco mantenimiento y presentan alta eficiencia, por medio de mecanismos naturales y altamente sostenibles Peña y Mara, (2004). Valledupar es ciudad pionera en el tratamiento de residuos líquidos, al ser la primera ciudad en la costa atlántica y una de las pocas ciudades en Colombia, que trata el $100 \%$ de las aguas residuales. El sistema está constituido por tres fases. Un tratamiento primario consistente en desarenación y cribado. Una segunda fase consistente en lagunas anaeróbicas seguidas de facultativas y un tratamiento terciario consistente en lagunas de maduración.

Los análisis de la calidad de las aguas están basados casi exclusivamente en los métodos fisicoquímicos y microbiológicos Chávez y Orantes, (2010). El uso de los macroinvertebrados acuáticos como indicadores de la calidad del agua está teniendo un auge importante en todo el mundo Prat et al., (2009). Los macroinvertebrados se han utilizado ampliamente como herramienta para determinar la calidad del agua Roldán, (1999); Fierro, et al., (2015) debido, entre otras razones, a que su taxonomía es relativamente sencilla, son abundantes y diversos, los materiales y métodos de muestreo están estandarizados y son de bajo costo y, además, responden a agentes contaminantes, lo que permite diagnosticar la calidad y el estado de conservación del cuerpo de agua en el que habitan Roldán, (1999). Los estudios de este tipo son escasos, siendo representativos los trabajos Raburu et al., (2017) donde se usaron macroinvertebrados acuáticos para evaluar rendimiento de los estanques de estabilización que tratan efluentes del procesamiento de la caña de azúcar y la melaza. Dada la falta de información sobre este tema en Colombia, el presente estudio tuvo como objetivo evaluar la eficiencia del sistema de tratamiento de las lagunas de estabilización El Salguero utilizando los macroinvertebrados acuáticos como bioindicadores, aplicando el índice biótico BMWP adaptado para Colombia por Roldan, (2003), además de los índices bióticos de riqueza alfa y beta, como línea de referencia para programas de monitorización de sistemas de lagunas de estabilización y con el fin de implementar estrategias de biomonitorización.

\section{MATERIALES Y METODOS}

Se describe el área de estudio, luego se describen los puntos de muestreo y la recolección de organismos, terminando con una explicación sobre el análisis de variables.

\section{Área de estudio}

El sistema de lagunas de estabilización El Salguero (73¹4'07.15' O' y $\left.10^{\circ} 23^{\prime} 16.65^{\prime \prime} \mathrm{N}\right)$, se encuentra ubicado en el costado sur de la cabecera municipal, en inmediaciones de la llamada curva del Salguero, a unos 9 kilómetros del casco urbano de la Ciudad de Valledupar y a 116 m.s.n.m; está constituida actualmente por una zona de cribado y desarenado, lagunas anaerobias, lagunas facultativas y de maduración (Figura 1).

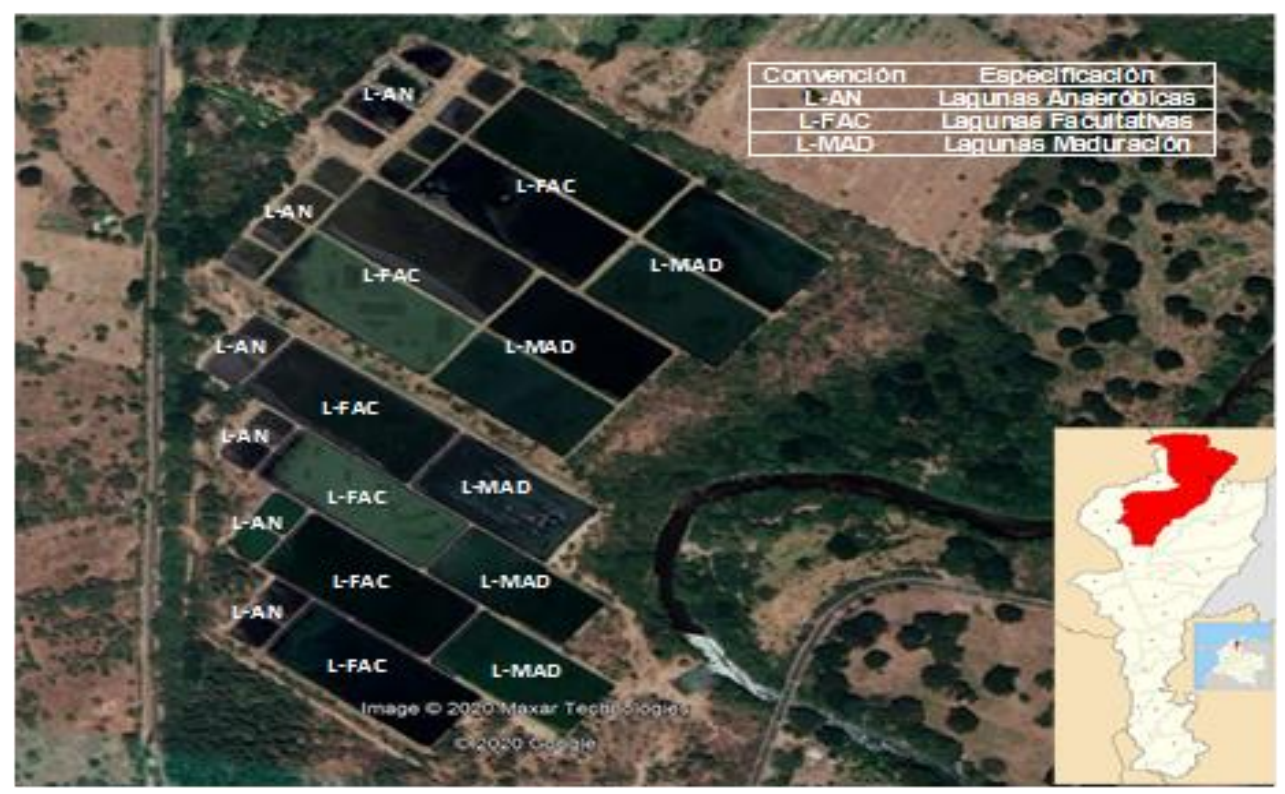

Fig. 1: Ubicación del área de estudio. 
Comprende una sección de 513 metros por 110 metros, para un área aproximada de 5,64 hectáreas netas, las cuales sumadas a las áreas libres alcanzan una extensión total de 6 hectáreas aproximadamente. El Star Salguero cuenta para su tratamiento con ocho módulos. Cada módulo consta de una estructura de reparto (Aforador de Caudal), un desarenador, tubería de entrega, dos lagunas anaeróbicas, una laguna facultativa y una laguna de maduración. Cada módulo consta de una estructura de tratamiento primario con una rejilla de cribado, posteriormente el agua atraviesa la unidad de desarenador compuesta por 8 módulos cada uno de los cuales presenta dos cámaras de flujo horizontal donde se precipitan las partículas más pesadas.

\section{Fase de Campo}

Se realizaron muestreos durante el mes de enero de 2018, cubriendo las 16 lagunas anaeróbicas, ocho facultativas y siete de maduración, para un total de 31 muestras. La recolecta de las muestras se realizó de forma directa con el uso de una red D-Net de $500 \mu \mathrm{m}$ de abertura de malla. La recolección de los macroinvertebrados se realizó en los microhábitats observados (vegetación y sedimento). Se hizo un arrastre con la red D-Net en los microhábitats identificados. La muestra fue colocada en bandejas con agua y se recolectaron los macroinvertebrados con la ayuda de pinzas entomológicas. Los macroinvertebrados fueron colocados en frascos rotulados con alcohol al 70\% y glicerina, para su posterior identificación. Los macroinvertebrados acuáticos fueron identificados hasta el nivel taxonómico de familia con base en las descripciones y claves taxonómicas de Roldán, (1988) y Domínguez y Fernández, (2009); cabe resaltar que la información disponible en Colombia sobre la taxonomía, la ecología y la bioindicación de la calidad del agua no es similar en todos los grupos. Ephemeroptera, Plecóptera y Trichoptera cuentan con mayor información que los demás taxones. Es importante, además, profundizar en el estudio de los anélidos, los moluscos y los dípteros, especialmente de la familia Chironomidae Roldan, (2016).

\section{Análisis de variables}

Se aplicaron los índices de diversidad alfa, Shannon-Wiener $\left(\mathrm{H}^{\prime}\right)$, Dominancia de Simpson ( $\left.\mathrm{D}^{\prime}\right)$, Riqueza de Margalef $\left(D_{M g}\right)$ y Equidad de Pielou $\left(J^{\prime}\right)$; para la diversidad beta se realizaron análisis de similitud de los puntos monitoreados considerando la presencia y ausencia de los taxones de macroinvertebrados acuáticos empleando el índice de Jaccard Magurran, (2004) y otro de similitud de las familias considerando su abundancia con el índice Bray \& Curtis. En ambos casos el dendrograma de similitud se construyó por el método de promedios no ponderados (UPGMA). Todos los análisis se realizaron con el programa Past $\vee 3.20$ y BioDiversity pro V 2.0. La evaluación de la calidad del agua en los tramos estudiados se realizó a través del cálculo del índice biológico BMWP/Col. En el cual se ordenaron las familias de macroinvertebrados en 10 niveles con las puntuaciones de 1 a 10, siendo 1 un número de mayor tolerancia y 10 de menor tolerancia Roldan, (2003), según ecuación 1.

$B M W P=T 1+T 2+T 3+\ldots T n$

Donde, $T$ es el nivel de tolerancia y el número corresponde a la familia, resultando una sumatoria de todas las familias que indican los niveles de calidad de agua.

\section{RESULTADOS Y DISCUSION}

Los resultados se presentan en tres subsecciones: i) caracterización biológica; ii) Índices ecológicos iii) puntaje BMWP/Col.

\section{Caracterización Biológica}

Como se observa en la tabla 1, se registró un total de 2619 macroinvertebrados acuáticos distribuidos en tres phylum, tres clases, cinco órdenes y 14 familias identificadas. El orden con mayor número de organismos fue Díptera con 2382 individuos que corresponden al 91\% de la muestra, los demás órdenes reportaron abundancias menores al 5\% (Figura 2). En cuanto a la riqueza, esta fue menor en las lagunas anaeróbicas y mayor en las lagunas de maduración, coincidiendo con estudios realizados por Figueroa et al., (2007), Olivares et al., (2012) y Madera et al., (2016) quienes observaron una disminución de la riqueza de especies de acuerdo al mayor grado de contaminación. La mayor cantidad de macroinvertebrados acuáticos fueron recolectados en las lagunas facultativas donde se obtuvieron un total de 1235 organismos que representaron el $47,2 \%$, seguido de las lagunas anaeróbicas con 1032 organismos para un $39,4 \%$ y por ultimo las lagunas de maduración reportaron la menor abundancia con 352 organismos que corresponden al $13,4 \%$ de la población total. En cuanto a composición, se obtuvo que tres familias son compartidas por los tres sistemas de lagunas, entre ellas Culicidae, Psychodidae y Ephydridae. Se presentó una familia exclusiva para las lagunas de facultativas que corresponde a Corixidae, para las lagunas de maduración se presentaron tres familias, incluyendo a Elmidae, Belostomatidae y Planorbidae. 


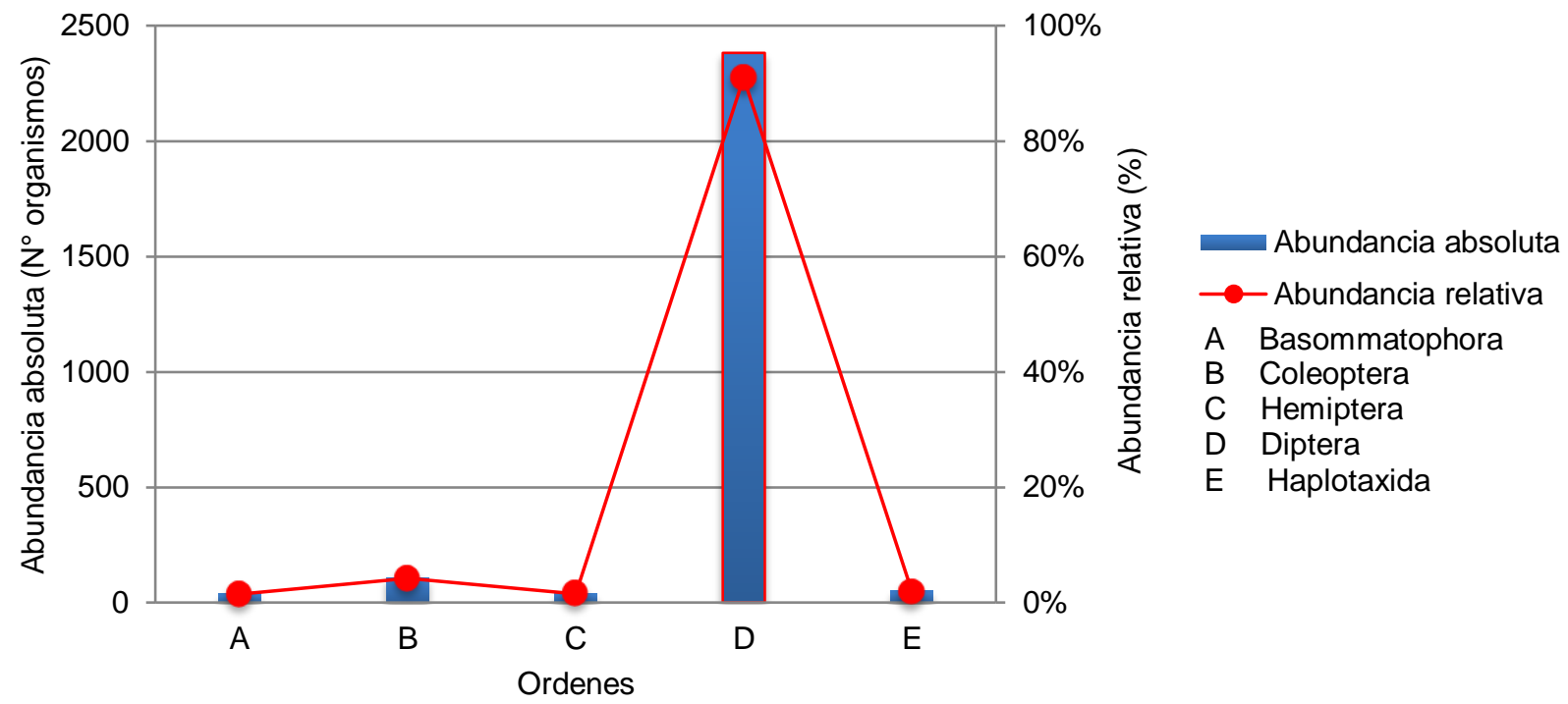

Fig. 2: Abundancia absoluta vs abundancia relativa de los órdenes de los macroinvertebrados acuáticos en el sistema de lagunas de estabilización El Salguero, enero 2018.

Tabla 1: Composición taxonómica de la comunidad de macroinvertebrados acuáticos registrados en el sistema de lagunas de estabilización El Salguero, enero 2018.

\begin{tabular}{|c|c|c|c|c|c|}
\hline Clase & Orden & Familia & $\begin{array}{l}\text { Lagunas } \\
\text { Anaeróbicas }\end{array}$ & $\begin{array}{l}\text { Lagunas } \\
\text { Facultativas }\end{array}$ & $\begin{array}{l}\text { Lagunas de } \\
\text { Maduración }\end{array}$ \\
\hline \multirow[t]{12}{*}{ Insecta } & \multirow[t]{6}{*}{ Diptera } & Culicidae & 752 & 577 & 125 \\
\hline & & Psychodidae & 97 & 110 & 27 \\
\hline & & Ephydridae & 77 & 46 & 25 \\
\hline & & Syrphidae & 54 & 21 & \\
\hline & & Stratiomyidae & 18 & & 9 \\
\hline & & Chironomidae & & 387 & 57 \\
\hline & \multirow[t]{4}{*}{ Coleoptera } & Hydrophiilidae & & 21 & 15 \\
\hline & & Dytiscidae & & 14 & 17 \\
\hline & & Noteridae & & 21 & 11 \\
\hline & & Elmidae & & & 10 \\
\hline & \multirow[t]{2}{*}{ Hemiptera } & Corixidae & & 22 & \\
\hline & & Belostomatidae & & & 18 \\
\hline Gastropoda & Basomatophora & Planorbidae & & & 38 \\
\hline Oligochaeta & Haplotaxida & Tubificidae & 34 & 16 & \\
\hline \multicolumn{3}{|l|}{ Total } & 1032 & 1235 & 352 \\
\hline \multicolumn{3}{|c|}{$\%$ Abundancia } & 39,4 & 47,2 & 13,4 \\
\hline
\end{tabular}

Los dípteros fueron los más diversos con 6 familias, dentro de las cuales la familia Culicidae fue la más abundante con 1454 organismos que corresponden al $56 \%$ de la muestra, seguido de la familia Chironomidae con 444 organismos que corresponden al 17\%, la abundancia y riqueza taxonómica de larvas de dípteros, se puede atribuir a las adaptaciones morfológicas (sifones y espiráculos para la toma de oxígeno), alimenticias (la mayoría son colectores y filtradores de materia fina, muy abundante en fitotelmata como producto de la degradación de la hojarasca) y reproductivas (la oviposición en el hábitat donde se desarrollará la larva junto con su alta capacidad de dispersión dada por el vuelo) (Ospina-Bautista et al. 2004); el orden Coleóptera fue el segundo más diverso con 4 familias, siendo la familia Hydrophilidae la más representativa con 36 organismos que corresponden al $1,4 \%$, le siguió el orden Hemíptera con dos familias Corixidae y Belostomatidae las cuales representaron el 0,8 y $0,7 \%$ respectivamente, por último los órdenes Basommatophora y Haplotaxida solo reportaron una familia para cada uno las cuales representaron el 1,5 y $2 \%$ respectivamente (Figura 3); resultados similares fueron observados en Mosquera et al, (2016) donde el 
orden Diptera fue el más abundante y diverso, representado en gran parte por la familia Culicidae, seguido por el orden Coleoptera, Raburu et al., (2017) demostró que los dípteros dominaron en las lagunas que tenían altas concentraciones de DBO5, DQO y nutrientes y Nuñez y Fragoso, (2019) reportaron el mayor número de taxones y abundancia de los dípteros, siéndola familia Chironomidae la más abundante en sedimentos con altas concentraciones de materia orgánica.

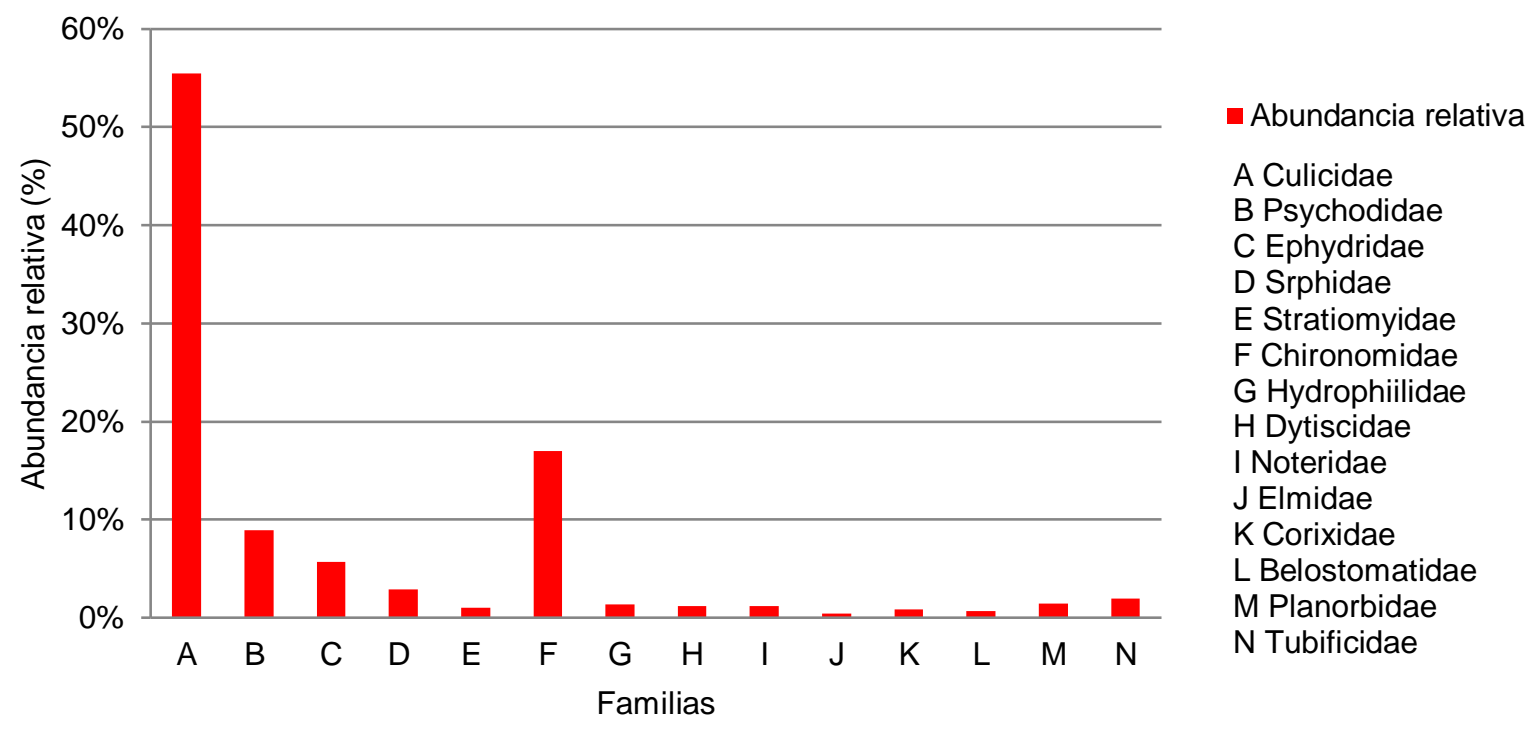

Fig. 3: Abundancia relativa de las familias de los macroinvertebrados acuáticos en el sistema de lagunas de estabilización El Salguero, enero 2018.

\section{Índices Ecológicos}

Los resultados de los índices ecológicos difieren entre los puntos de muestreo (Tabla 2). La mayor diversidad de Shannon-Weaver se presentó en las lagunas de maduración con un valor de 2,02 bits, seguida de las lagunas facultativas con 1,44 bits, las lagunas anaeróbicas registraron la diversidad más baja con 0,98 bits, según Roldan y Ramírez, (2008) la clasificación de la calidad del agua basado en el índice de ShannonWeaver, las lagunas anaeróbicas y facultativas se encuentran en el rango entre 0,0 y 1,5 indicando que son aguas muy contaminadas, las lagunas de maduración se encuentran en el rango entre 1,5 y 3,0 indicando que son aguas medianamente contaminadas. La riqueza de Margalef registro una baja diversidad en todo el sistema de lagunas de estabilización, valores por debajo de 2 son considerados como baja diversidad Margalef, (1998). La dominancia presento un valor relativamente alto en las lagunas anaeróbicas, puede estar relacionado con la alta densidad que mostro la familia Culicidae la cual represento el $73 \%$ de los organismos encontrados, en cuanto a la equidad se observa que las lagunas de maduración presentaron el valor más alto, indicando una mejor distribución de las especies, estando relacionada con la mayor diversidad y riqueza, así como la menor dominancia.

Tabla 2: Valores de los índices de diversidad alfa en el sistema de lagunas de estabilización El Salguero, enero 2018.

\begin{tabular}{|l|l|l|l|l|}
\hline Puntos de muestreo & Shannon $\left(H^{\prime}\right)$ & Margalef $\left(D_{M g}\right)$ & Dominancia $(D)$ & Equidad $\left(J^{\prime}\right)$ \\
\hline Lagunas Anaeróbicas & 0,98 & 0,72 & 0,55 & 0,55 \\
\hline Lagunas Facultativas & 1,44 & 1,26 & 0,32 & 0,62 \\
\hline Lagunas de Maduración & 2,02 & 1,7 & 0,18 & 0,84 \\
\hline
\end{tabular}

El análisis de similitud entre las diferentes lagunas de estabilización por la presencia-ausencia de los taxones permitió la identificación de un agrupamiento entre las lagunas facultativas y de maduración con un $50 \%$ de similitud, las lagunas anaeróbicas se agrupan con las lagunas facultativas mostrando una similitud mayor al 45\% (Figura 4). El análisis de similitud de Bray-Curtis mostró las asociaciones entre los diferentes taxones y evidenció cuatro grupos (I, II, III, y IV) (Figura 5). El grupo I lo integran las familias Culicidae y Chironomidae las cuales fueron las de mayor abundancia de todo el estudio. El grupo II incluye a las familias Psychodidae, 
Ephydridae, Syrphidae y Tubificidae, estas familias presentaron una abundancia media. El grupo III está representado por las familias Hydrophiilidae, Noteridae, Dytiscidae y Corixidae que tienen alta frecuencia de ocurrencia, pero cuya abundancia no fue alta. El último grupo, el IV se conformó por las familias Elmidae y Belostomatidae, caracterizados por su escasa abundancia y ocurrencia ya que fueron taxones poco representados en el sistema de lagunas y con una distribución restrictiva. La familia Stratiomyidae se muestra aislada de las demás familias, posiblemente por ser la menos representativa dentro del orden de los dípteros, con respecto a Planorbidae, se observa que se asocia a los grupos III y IV los cuales fueron los de menor densidad poblacional.

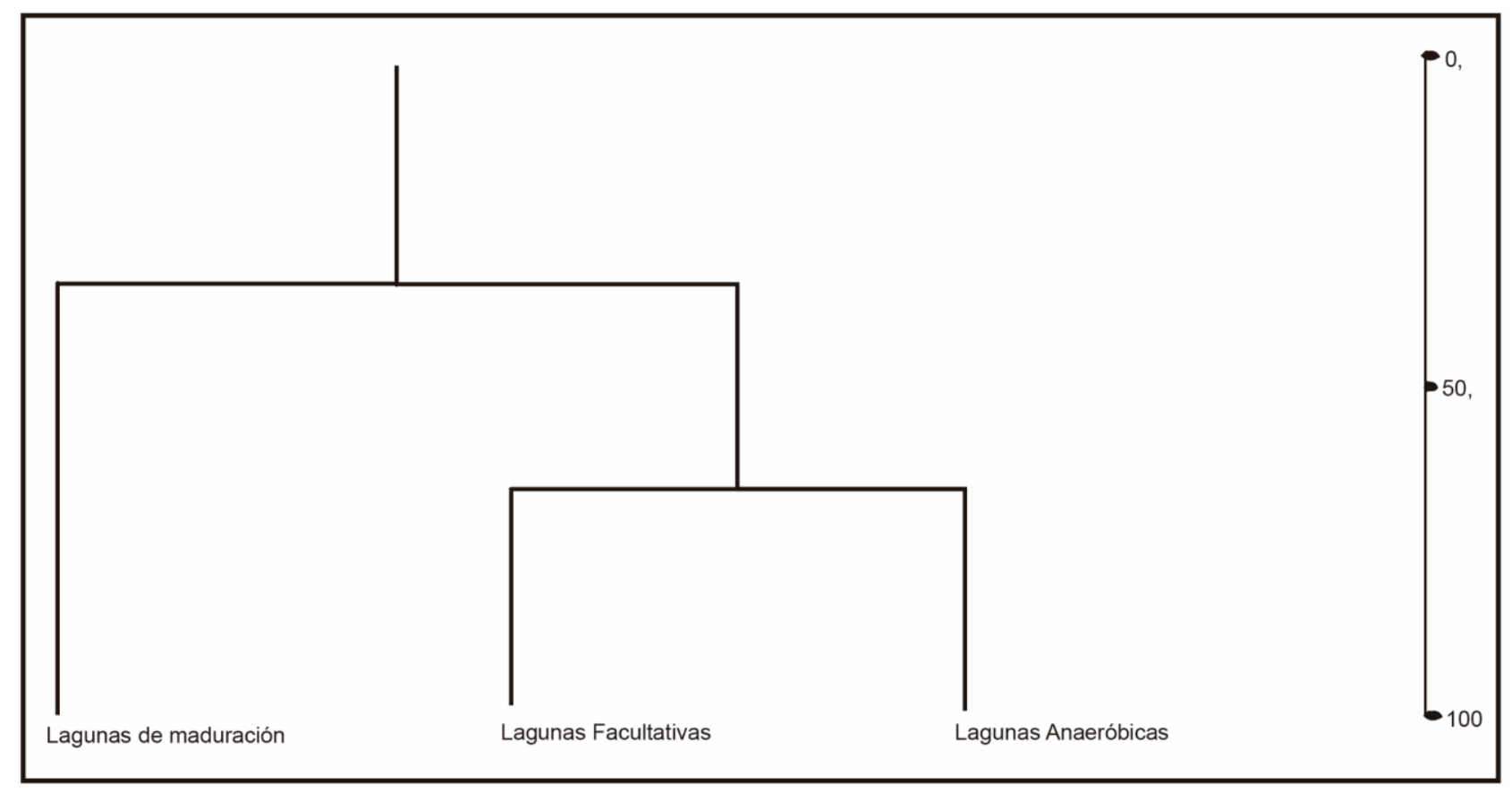

Fig. 4: Dendrograma de similitud para los puntos de muestreo basado en el Índice de Jaccard en el sistema de lagunas de estabilización El Salguero, enero 2018.

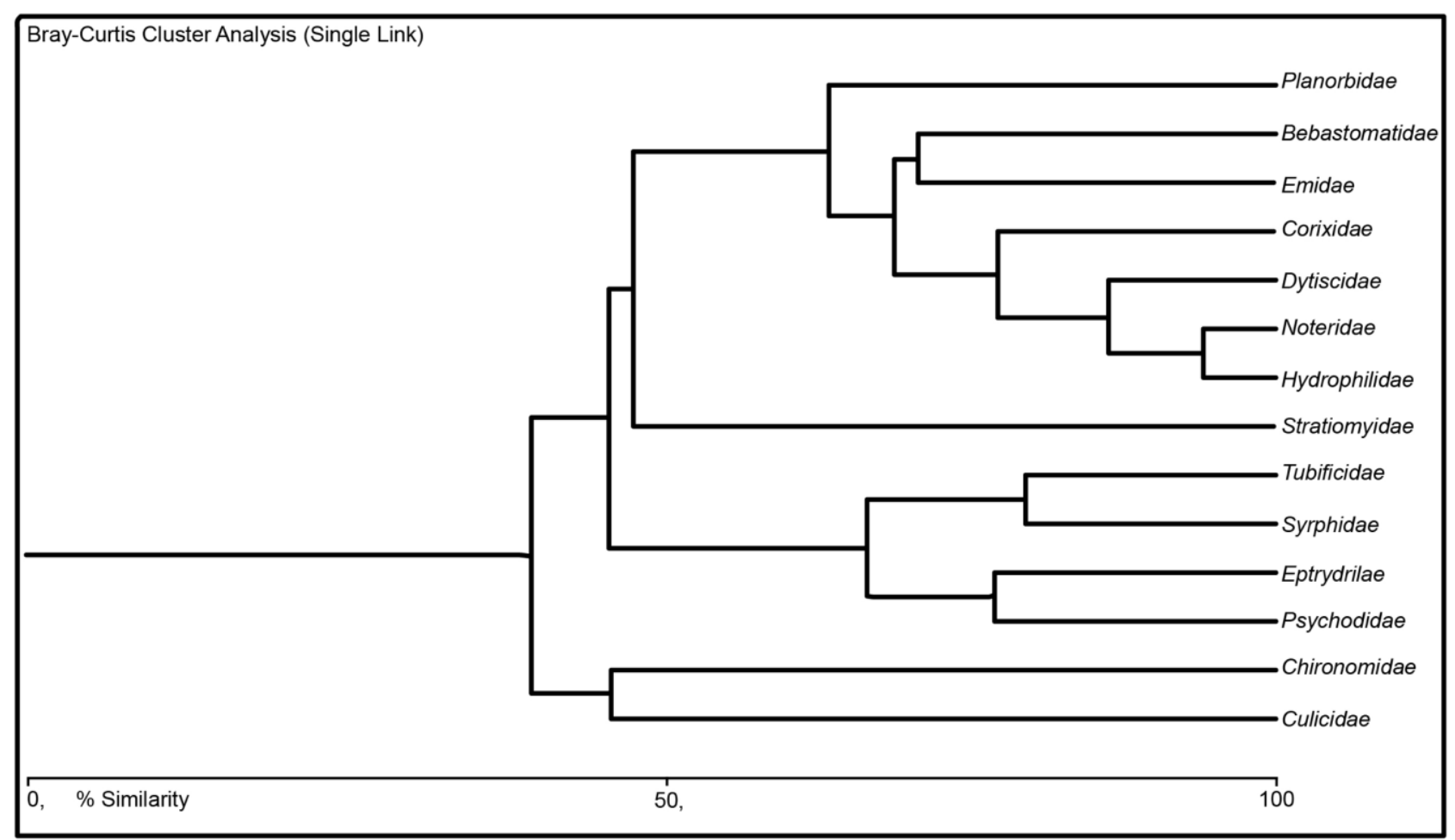

Fig. 5: Dendrograma de similitud para las familias de macroinvertebrados acuáticos basado en el Índice de Bray-Curtis en el sistema de lagunas de estabilización El Salguero, enero 2018. 


\section{Puntaje BMWP/Col}

El análisis de los resultados del índice BMWP/Col (Tabla 3), fueron registrados para cada sistema de lagunas de estabilización. Se presentaron una cantidad considerable de macroinvertebrados acuáticos indicadores de mala calidad de aguas, como era de esperarse en aguas contaminadas; según el índice BMWP/Col las lagunas anaeróbicas se ubican en la clase V, de calidad muy crítica, cuya característica son de aguas fuertemente contaminadas; la presencia de dípteros de las familias Syrphidae, Culicidae, Psychodidae, Stratiomyidae y un porcentaje significativo de oligoquetos pertenecientes a la familia Tubificidae los cuales son indicadores de aguas con alto grado de contaminación por materia orgánica; las lagunas facultativas se ubican en la clase IV, de calidad critica, indicando aguas muy contaminadas, aun así se presentaron algunas familias indicadoras de aguas medianamente contaminadas como las familias Corixidae y Dytiscidae, pero predominaron organismos indicadores de aguas contaminadas como Syrphidae, Culicidae, Psychodidae y Stratiomyidae; para las lagunas de maduración se reportaron familias indicadoras de aguas contaminadas pertenecientes al orden díptera ya mencionadas, así como también organismos indicadores de aguas moderadamente contaminadas como algunos coleópteros y la presencia de hemípteros y del orden Basommatophora, ubicando las lagunas en la clase III, de calidad dudosa, indicando aguas moderadamente contaminadas.

Tabla 3: Valores del índice BMWP/Col registrados en el sistema de lagunas de estabilización El Salguero, enero 2018.

\begin{tabular}{|l|l|l|l|l|}
\hline \multicolumn{1}{|c|}{ Punto de muestreo } & \multicolumn{1}{c|}{$\begin{array}{c}\text { Valor } \\
\text { BMWP/Col }\end{array}$} & \multicolumn{1}{c|}{ Clase } & \multicolumn{1}{c|}{ Calidad } & \multicolumn{1}{c|}{ Significado } \\
\hline Lagunas Anaeróbicas & 14 & V & Muy Critica & Aguas fuertemente contaminadas \\
\hline Lagunas Facultativas & 33 & IV & Critica & Aguas muy contaminadas \\
\hline Lagunas de Maduración & 47 & III & Dudosa & Aguas moderadamente contaminadas \\
\hline
\end{tabular}

La abundancia de los quironómidos y culícidos (Díptera) en las lagunas estudiadas se relaciona con su capacidad para tolerar altos grados de contaminación del agua. Estas larvas viven en aguas estancadas o de corriente, resisten altos grados de contaminación y son indicadores de agua de mala calidad Gamboa et al., (2008). Las comunidades naturales se caracterizan por tener varias familias que los representan, pero pocos individuos por familias. Sin embargo, en los ambientes donde existe un mayor grado de contaminación, las familias se reducen, pero aumentan la cantidad de los individuos por familia Roldan, (1999). Basado en esto se puede decir que las lagunas con mayor grado de contaminación por la presencia de la materia orgánica son las anaeróbicas. Los cambios en la sedimentación y en la disminución del oxígeno disuelto suelen ser evidencia de una mayor presencia de la materia orgánica, lo que ocasiona una reducción de los órdenes de macroinvertebrados, lo que provoca que las familias que no son tolerantes a estos cambios vayan desapareciendo, por lo tanto, la diversidad Muñoz et al., (2001).

En relación a las lagunas de maduración donde el grado de contaminación es menor, la abundancia de los individuos fue baja, no obstante, el número de taxa fue alta, que es lo que se espera encontrar en los lugares no contaminados o con poca contaminación Roldán, (1999). Al ser las últimas lagunas de todo el sistema y presentar las mejores condiciones, se espera que el proceso de depuración del agua residual haya sido eficiente. La eficiencia de la depuración del agua residual también afecta la comunidad de los macroinvertebrados, por lo que conforme se va eliminando la materia orgánica de las lagunas anaeróbicas y facultativas y mejoran las condiciones fisicoquímicas, la abundancia de las familias de los macroinvertebrados aumenta y se reduce el número de individuos por familia.

\section{CONCLUSIONES}

Los estudios a nivel de campo y laboratorio permiten afirmar lo siguiente: 1) La mayor diversidad de los macroinvertebrados acuáticos se obtuvo en las lagunas de maduración, siendo el orden Diptera los que tienen mayor presencia de individuos en cada fase de tratamiento; 2) El índice BMWP/Col en base a los macroinvertebrados acuáticos, define las aguas de las lagunas anaeróbicas como fuertemente contaminadas de calidad muy crítica, las lagunas facultativas como aguas muy contaminadas de calidad dudosa y las lagunas de maduración como aguas moderadamente contaminadas de calidad aceptable; 3) A medida que las condiciones ambientales son favorables y la contaminación del agua se reduce, aumenta el número de los taxa y disminuye el número de organismos; 4) Los macroinvertebrados como bioindicadores proveen información de la calidad del agua y la presencia de la contaminación que se relaciona directamente con los órdenes encontrados.

\section{REFERENCIAS}

Chávez, J.M., y Orantes, E.D., Reconocimiento de las comunidades de macroinvertebrados acuáticos como alternativa para determinar la calidad del agua del Río Sensunapán, Departamento de Sonsonate, El Salvador, C.A. Tesis Ing. Agrónomo. San Salvador, El Salvador, Universidad de El Salvador, 113 (2010). 
Domínguez, E., y Fernández, H., Macroinvertebrados bentónicos sudamericanos, sistemática y taxonomía. Primera edición, Tucuman. Fundación Miguel Lillo, 1-652 (2009).

Fierro, P., Bertran, C., y otros seis autores, Landscape composition as a determinant of diversity and functional feeding groups of aquatic macroinvertebrates in southern river of the Araucanía, DOI: 10.3856/vol43-issue1-fulltext-16, Chile, Latin American Journal of Aquatic Research. 43 (1): 186-200 (2015) .

Figueroa, R., Palma, A., y otros dos autores, Análisis comparativo de índices bióticos utilizados en la evaluación de la calidad de las aguas en un río mediterráneo de Chile: Río Chillán, VIII Región, http://dx.doi.org/10.4067/S0716078X2007000200008, Rev. Chilena de Historia Natural, 80 (8), 227-242 (2007).

Gamboa, M., Ryes, R., y Arrivilla, J., Macroinvertebrados bentónicos como bioindicadores de salud ambiental, Malariología y Salud Ambiental 48(2), 109-108 (2008).

Jimoh, T.A., Keshinro, M.O., Cowan, K.A., MicroalgaL-Bacterial Flocs and Extracellular Polymeric Substances: Two Essential and Valuable Products of Integrated Algal Pond Systems, https://doi.org/10.1007/s11270-019-4148-3, Water Air, and Soil Pollution, 230 (4), 95, (2019).

Madera, L., Angulo. L., y otros dos autores, Evaluación de la Calidad del Agua en Algunos Puntos Afluentes del río Cesar (Colombia) utilizando Macroinvertebrados Acuáticos como Bioindicadores de Contaminación, doi: 10.4067/S071807642016000400011, Rev. Información Tecnológica, 27(4), 103-110 (2016).

Magurran, A.E., Measuring biological diversity, Blackwell Publishing, Oxford, 101-130 (2004).

Mambo, P. M., Westensee, D. K., y otros dos autores, Operation of an integrated algae pond system for the treatment of municipal sewage, doi: 10.2166/wst.2014.187, Water Science and Technology, 69(12), 2554-2561. (2014).

Margalef, R., Ecología. Barcelona: Ediciones Omega, SA; 320 (1998).

Mosquera, M., Gómez, M.A., Gonzales, M.B., Diversidad de macroinvertebrados acuáticos asociados con Bromeliaceae en una zona de bosque pluvial tropical, Chocó, Colombia, DOI: 10.18636/bioneotropical.v6i2.350, Rev. Biodivers. Neotrop, 6 (2): 147-53 (2016).

Muñoz, E., Mendoza, G., y Valdovinos, C., Evaluación rápida de la biodiversidad en cinco sistemas lenticos de Chile central, macroinvertebrados bentónicos, http://dx.doi.org/10.4067/S0717-65382001000200009, Gayana. 65(2), 173-180 (2001).

Núñez, J., y Fragoso, P., Uso de macroinvertebrados acuáticos como bioindicadores de contaminación del agua de la Ciénaga Mata de Palma (Colombia), doi: 10.4067/S0718-07642019000500319, Inf. Tecnol., 30(5), 319 -330 (2019).

Olivares, C.G., Naranjo L.C., y otros dos autores, Valoración de la calidad del agua del río San Juan de Santiago de Cuba asociado a un foco de contaminación industrial, Rev. Ciencia en su PC, 4, 99-111 (2012).

Ospina-Bautista, F., Estévez, V., Betancur, J., Realpe, E., Invertebrados acuáticos asociados a Tillandsia turneri Baker (Bromeliaceae) en un bosque altoandino (Cundinamarca, Colombia), ISSN electrónico 1900-1649, Acta Zool Mex. 20 (1): 153-66 (2004).

Peña, M., y Mara, D., Waste Stabilization Ponds. The Netherlands: IRC International Water and Sanitation Centre, (2004).

Prat, N., Ríos, B., Acosta, R., y Rieradevall, M., Los macroinvertebrados como indicadores de calidad de las aguas. En: Domínguez, E., y Fernández, H.R., Macroinvertebrados bentónicos sudamericanos. Sistemática y biología. Fundación Miguel Lillo, Tucumán, Argentina. 26 (2009).

Raburu, P.O., Masese, F.O., y Tonderski, K.S., Use of macroinvertebrate assemblages for assessing performance of stabilization ponds treating effluents from sugarcane and molasses processing, https://doi.org/10.1007/s10661-017-57869, Environ Monit Assess, 189, 79 (2017).

Roldán, G., Guía para el estudio de los macroinvertebrados acuáticos del departamento de Antioquia. FEN-Colciencias, Bogotá, 217 (1988).

Roldán, G., Los macroinvertebrados y valor como indicadores de la calidad de agua, doi: http://dx.doi.org/10.18257/raccefyn.335, Revista Académica Colombia, 23(88), 375-387 (1999).

Roldán, G., Bioindicación de la calidad del agua en Colombia. Uso del método BMWP/Col. Editorial Universidad de Antioquia, Medellín, 170 (2003).

Roldán, G., y Ramírez, J., Fundamentos de Limnología Neotropical. Editorial Universidad de Antioquia, Segunda Edición. Medellin-Colombia, 180-238 (2008).

Roldan, G., Los macroinvertebrados como bioindicadores de la calidad del agua: cuatro décadas de desarrollo en Colombia y Latinoamérica, https://doi.org/10.18257/raccefyn.335, Revista De La Academia Colombiana De Ciencias Exactas, Físicas Y Naturales, 40(155), 254-274 (2016).

Spellman, F., Handbook of Water and Wastewater Treatment Plant Operations. 2ª Ed. Boca Raton, FL: CRC Press, (2009). 